\title{
Influence of Germanium Interdiffusion on the Morphological Evolution of $\Sigma 3$ Grain Boundaries in Gold Thin Films
}

\author{
T. Radetic and U. Dahmen \\ National Center for Electron Microscopy, MSD, LBNL, Berkeley, 94720 CA, USA
}

Grain boundaries are characterized by $\mathrm{f}$ ive degrees of freedom: three variables characterize misorientation and two parameters determine inclination (grain boundary plane). For a defined misorientation, particular grain boundary planes may have lower energy then others, resulting in interfacial anisotropy that can be described by a Wulff shape. If the Wulff shape has forbidden orientations, grain boundaries with these inclinations will break into facets of neighboring orientations. A well-known example is the $\Sigma 3$ misorientation where the $\{111\}$ and boundary $\{112\}$ inclinations have the lowest energy, and faceting is commonly observed. For instance, faceting of $\Sigma 3$ grain boundaries in mazed bicrystals of Au was studied recently by Hetherington et al. [1] using conventional and high resolution transmission electron microscopy.

$\{111\}$ mazed bicrystal thin films of gold were grown epitaxially on germanium substrates. The morphology of the films is characterized by a random distribution of many $\{111\}$ grains in only two orientations that are related to each other by a symmetry operation of the substrate surface. In $\{111\}$ mazed bicrystals, the two orientations are twin related: one is characterized by ABCA and the other by ACBA stacking sequence of close-packed planes. The $\{111\}$ coherent twin plane is parallel to the substrate surface and the grain boundaries perpendicular to the surface are incoherent twin boundaries. It was reported that vertical grain boundary inclinations show strong anisotropy with predominant $\{112\}$ fac ets [1]. Recently, the morphological evolution of $\{111\}$ Au mazed bicrystals during in-situ annealing was studied by Medlin who reported coarsening of $\{112\}$ facets [2].

In this work, thin films $10-200 \mathrm{~nm}$ thick were grown by physical vapor deposition on $\{111\}$ Ge substrates at $280^{\circ} \mathrm{C}$ followed by annealing at $200^{\circ} \mathrm{C}$ to relieve stress. TEM specimens were prepared by two methods: chemical etching of the Ge substrate and ion -milling from the substrate side. When Ge is chemically removed, the free standing Au film free of Ge was put on a gold grid; similar to the procedure followed by [1,2]. During ion -milling from the substrate side, Ge was removed from the central area of the specimen leaving an electron transparent Au film. However, the film was still surrounded and supported by the substrate [3].

Study of the evolution of the film morphology during in -situ annealing revealed opposite behavior for specimens prepared by the different methods. In the case of free standing films, migration of ledges and coarsening of facets was observed (Fig. 1) as reported by Medlin [2]. The increase in the anisotropy of grain boundaries during annealing has been characterized quantitatively using rose plots, and the results show that all grain boundary planes adopt $\{112\}$ inclination s. Ion -milled samples exhibit fine scale faceting before annealing (Fig. 2a), indistinguishable from the freestanding film (Fig. 1a). However, during annealing and grain growth, these facets do not coarsen. Instead, grain boundaries become rounded and more isotropic (Fig. 2b). This difference in behavior is attributed to interdiffusion of Ge into the Au film and its influence on grain boundary structure. It has been reported that segregation of $\mathrm{Bi}$ in $\mathrm{Cu}$ grain boundaries causes faceting [4]. Apparently, Ge in Au leads to the opposite effect by eliminating faceting and resulting in a more isotropic grain morphology.

References

[1] C. J. D. Hetherington, et al, Mat. Res. Soc. Symp. Proc. 466 (1997) 215.

[2] D. L. Medlin and G. Lucadamo, Mat. Res. Soc. Symp. Proc. 652, (2001).

[3] T. Radetic and U. Dahmen, Mat. Res. Soc. Symp. Proc. 648, (2001).

[4] T. G. Ference and R. W. Balluffi, Scripta Metall 22, (1988) 1929. 
[5] This work was supported by the Director, Office of Basic Energy Sciences, Materials Science Division, US Department of Energy, under contract DE-AC3-76SF00098.

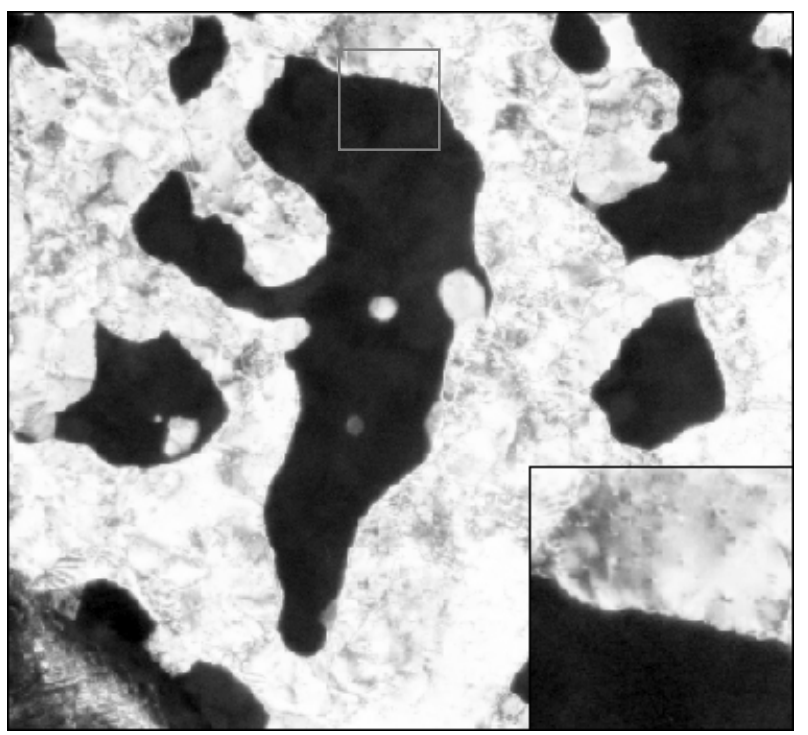

FIG. 1a. Morphology of a freestanding gold film before annealing. Fine corrugations due to faceting are observed in the insert.

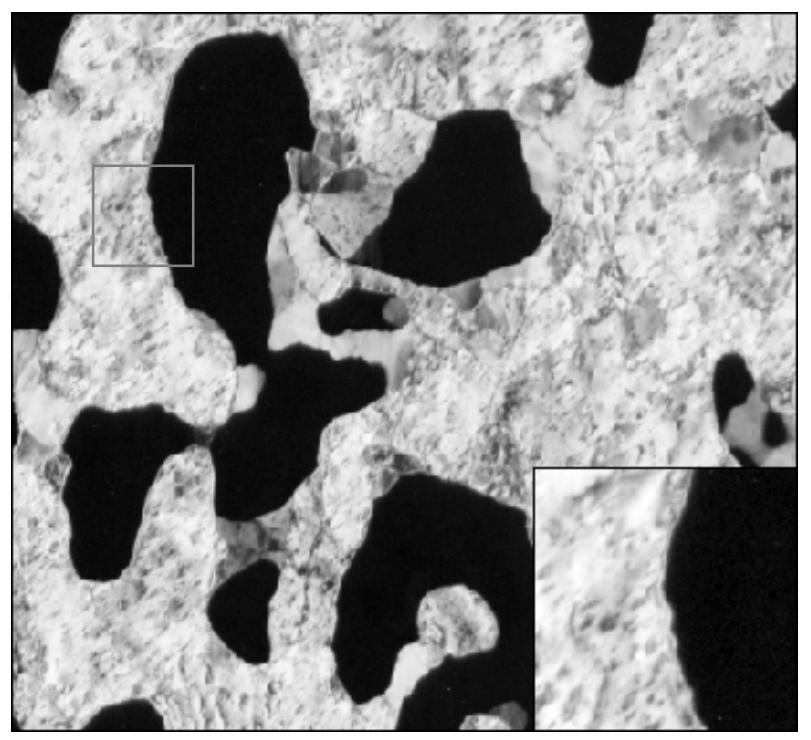

FIG. 2a. Morphology of an ion -milled gold film on Ge substrate before annealing. Fine corrugations due to faceting are observed in the insert.

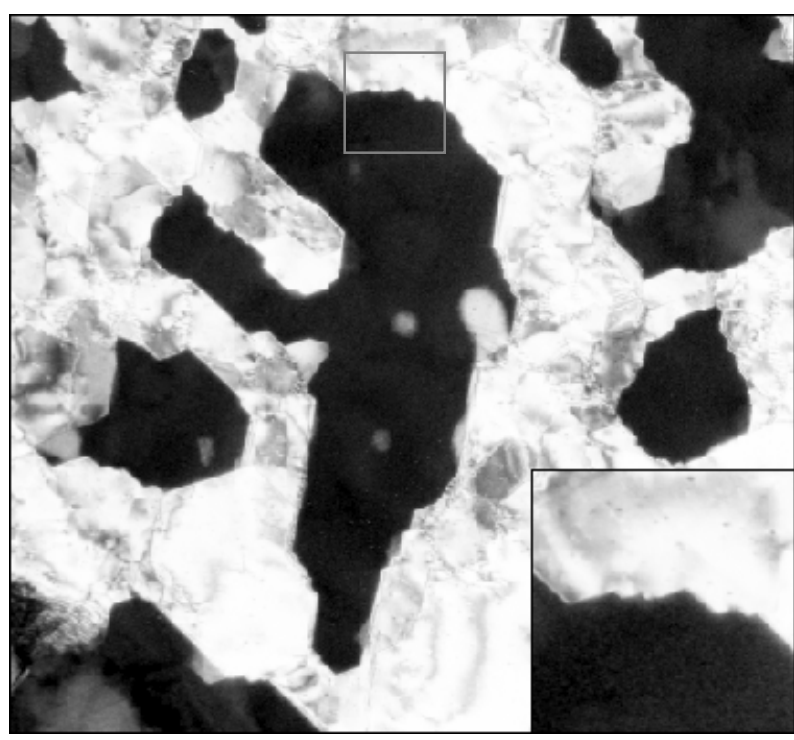

FIG. 1b. The same freestanding gold film after annealing for $30 \mathrm{~min}$ at $310^{\circ} \mathrm{C}$. Coarsening of the facets is apparent (see insert).

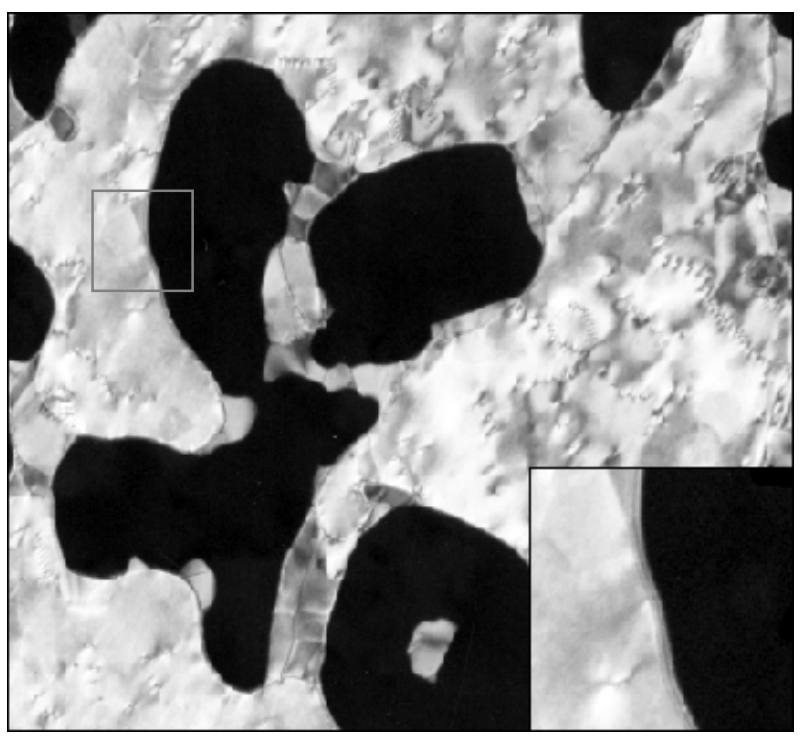

FIG. 2b. The same ion -milled gold film after annealing for $30 \mathrm{~min}$ at $310^{\circ} \mathrm{C}$. The grain morphology has become rounded and facets have disappeared (see insert). 